\title{
Behavioral estimates of interhemispheric transmission time and the signal detection method: A reappraisal
}

\author{
MARC BRYSBAERT \\ University of Leuven, Leuven, Belgium
}

\begin{abstract}
On the basis of a review of the literature, Bashore (1981) concluded that only simple reaction time experiments with manual responses yielded consistent behavioral estimates of interhemispheric transmission time. A closer look at the data, however, revealed that these experiments were the only ones in which large numbers of observations were invariably obtained from many subjects. To investigate whether the methodological flaw was the origin of Bashore's conclusion, two experiments were run in which subjects had to react to lateralized light flashes. The first experiment dealt with manual reactions, the second with verbal reactions. Each experiment included a condition without catch trials (i.e., simple reaction time) and two conditions with catch trials. Catch trials were trials in which no stimulus was given and in which the response was to be withheld. Both experiments returned consistent estimates of interhemispheric transmission time in the range of 2-3 msec. No differences were found between the simple reaction time condition and the signal detection conditions with catch trials. Data were analyzed according to the variable criterion theory. This showed that the effect of catch trials, as well as the effect of interhemispheric transmission, was situated at the height of the detection criterion, and not in the rate of the information transmission.
\end{abstract}

Since the original work of Poffenberger (1912), reaction times (RTs) have frequently been used to investigate the nature of interhemispheric transfer (IHT) of visual information in normal humans. The task is simple: Stimuli are presented to the left or right of fixation, and the subject has to react as soon as the stimulus appears. The reactions involve unilateral or bilateral limb movements, or voice responses. The most popular method consists of unilateral finger movements in response to light flashes (for reviews, see Bashore, 1981, and Marzi, Bisiacchi, \& Nicoletti, 1991). The subject is required to press on a key with a finger of the left or the right hand as soon as the light flash appears. Over a series of $16 \mathrm{ex}-$ periments, it has been shown that responses are consistently faster when subjects are allowed to react with the right hand on stimuli presented to the right of fixation, and with the left hand on stimuli presented to the left of fixation (Marzi et al., 1991). RTs are about $3.8 \mathrm{msec}$ (range, 1-10 msec) longer when subjects have to react with the right hand to light flashes on the left, or with the left hand to light flashes on the right. The difference is assumed to be due to the fact that in the latter conditions (also called the crossed conditions), stimulus perception and reaction control are mediated by different cerebral hemispheres, whereas in the former conditions (the uncrossed conditions), perception and reaction are

The author is a research associate of the Belgian National Fund for Scientific Research. He would like to thank Catherine Meyers, who ran Experiment 1. Correspondence should be addressed to M. Brysbaert, Department of Psychology, University of Leuven, B-3000 Leuven, Belgium (e-mail: fpaas02@blekul11.bitnet). governed by the same hemisphere, so that no IHT of information is needed. Further evidence for the claim that the difference between crossed and uncrossed responses is a measure of interhemispheric transfer time (IHTT) comes from the finding that the difference is larger for subjects with agenesis of the corpus callosum $(M=17.7$ msec) and for subjects with section of the corpus callosum $(M=54.8 \mathrm{msec}$ ) (Clarke \& Zaidel, 1989; Marzi et al., 1991).

Virtually all IHTT studies hitherto have made use of the simple reaction time (SRT) paradigm. This paradigm consists of a single response made to a single stimulus presented on each trial. The preference for the SRT design with manual reactions stems from an influential review by Bashore (1981), who noted that only simple visuomotor RT tasks provide reliable estimates of IHTT that closely approximate electrophysical measures. Table 1 is a partial replication of Bashore's first table, in which he compared IHTT estimates obtained in SRT designs and in signal detection (SD) designs (termed stimulus detection designs by Bashore). Both paradigms are identical, except that SD experiments contain catch trials. Catch trials are trials in which no stimulus is given and in which the response must be withheld. Although the difference between the paradigms is small, the table clearly illustrates that they do not provide the same estimates of IHTT: SRT experiments return a consistent estimate in the order of $2 \mathrm{msec}$, whereas SD experiments yield IHTT measures as divergent as 5 and $28 \mathrm{msec}$. This comparison made Bashore (1981) conclude that "until careful parametric analyses are completed, meaningful conclusions about the sources of IHTT variability and 
Table 1

Reaction Time (RT) Studies of Interhemispheric

Transmission Time (IHTT) Based on Unimanual Reactions to Lateralized Light Flashes

\begin{tabular}{|c|c|c|c|c|c|}
\hline Source & $N_{\text {subj }}$ & $N_{\text {obsisubj }}$ & $\mathrm{RT}_{\text {contra }}$ & $\mathrm{RT}_{\mathrm{ipsi}}$ & IHTT (msec) \\
\hline \multicolumn{6}{|c|}{ Simple RT } \\
\hline Poffenberger, 1912 & $\mathrm{IR} / 1 \mathrm{~L}^{\mathrm{a}}$ & 4,000 & 199.6 & 195.4 & $4.2^{\mathrm{b}}$ \\
\hline Smith, 1938 & $11 \mathrm{R} / 1 \mathrm{~L}$ & 800 & 250.0 & 249.5 & 0.5 \\
\hline Jeeves, 1969 & $10 \mathrm{R}$ & 600 & 229.7 & 226.9 & 2.8 \\
\hline Berlucchi et al., 1971 & $14 \mathrm{R}$ & 1,180 & 247.3 & 244.9 & 2.4 \\
\hline Anzola et al., 1977 & $16 \mathrm{R}$ & 1,460 & $206.7^{\mathrm{c}}$ & 205.3 & 1.4 \\
\hline Berlucchi et al., 1977 & $16 \mathrm{R}$ & 960 & 190.9 & 188.4 & 2.5 \\
\hline DiStefano et al., 1980 & $12 \mathrm{R}$ & 480 & 199.5 & 197.2 & 2.3 \\
\hline \multicolumn{6}{|c|}{ Signal Detection } \\
\hline Kleinman et al., 1976 & $33 \mathrm{R}$ & $30 S / 15 C^{d}$ & 307.0 & 302.0 & $5.0^{\mathrm{e}}$ \\
\hline Swanson et al., 1978 & $12 ?$ & ? & ? & ? & 28.5 \\
\hline Moscovitch \& Smith, 1979 & $12 R^{f}$ & $200 \mathrm{~S} / 200 \mathrm{C}$ & 274.1 & 264.8 & 9.3 \\
\hline Smith \& Moscovitch, 1979 & $15 / 7 \mathrm{R}^{\mathrm{f}, \mathrm{g}}$ & $100 \mathrm{~S} / 100 \mathrm{C}$ & 293.5 & 269.5 & 24.0 \\
\hline
\end{tabular}

Note-This is a partial replica of Table 1 in Bashore (1981), in which the distinction was made between simple RT studies and signal detection studies. (From "Vocal and Manual Reaction Time Estimates of Interhemispheric Transmission Time," by T. R. Bashore, 1981, Psychological Bulletin, 89, 352-368. Copyright 1981 by the American Psychological Association. Adapted by permission). ${ }^{\mathrm{a}} \mathrm{R}$, righthanded; L, lefthanded. 'Value lower than the one usually reported (e.g., Bashore, 1981), because in his discussion Poffenberger excluded the data of $45^{\circ}$ eccentricity which he found too "unreliable" (read "small"). 'Data reported by Marzi et al. (1991); only graphs were given in the original manuscript. ${ }^{\mathrm{d}} \mathrm{S}$, stimulus trials; $\mathrm{C}$, catch trials. ${ }^{\mathrm{e}}$ Estimate not obtained by full crossing of hand and visual half-field; only data with the right

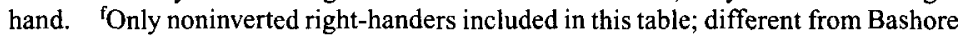
(1981). BData obtained in two different experiments: Experiment 1, 15 subjects responded with the left hand; Experiment 2, 7 of the original subjects responded with the right hand.

accurate estimates of IHTT with stimulus [signal] detection cannot be made" (p. 362).

Surprisingly, Bashore's review did not lead to the parametric analyses of SD studies that he had recommended; rather, the effect was that authors interested in IHTT no longer used SD designs to investigate the problem but confined themselves to SRT experiments (see Marzi et al., 1991, for a review). In this paper, I attempt to improve upon the situation by making a careful comparison of SRT and SD data that have been obtained under the same conditions.

Before we examine the empirical data, it may be interesting to inquire into the part of the process that is affected by the introduction of catch trials and how this may increase IHTT. As a guiding principle, I will make use of a simple mathematical model (i.e., the variable criterion theory of Grice, 1968) that has been proposed for the description of RT data (see, e.g., Luce, 1986, pp. 82-87). The model essentially consists of two parts: (1) stimulus information that accumulates in time, and (2) a criterion that must be reached before a response is emitted. The information accumulation is thought to be linear from an "irreducible minimum" $\left(R_{0}\right)$, with a slope that is proportional to the stimulus intensity. ${ }^{1}$ The detection criterion is assumed to be variable and experimentally manipulable. A schematic representation of the model is given in Figure 1. It displays the information accumulation (on the ordinate) for two different stimuli (a weak and a strong one) and two detection criteria that must be reached (a low and a high one). The intersection between the information and the detection line indicates the mean time (on the abscissa) needed in order to respond in a particular condition. Because the time difference between the weak and the strong stimulus is larger for the high-criterion condition than for the low-criterion condition, the model essentially predicts an interaction between stimulus intensity and criterion level. The introduction of catch trials most likely affects the detection criterion (see notes 3 and 7): More information must be collected before a response can be initiated if the answer is to be withheld on certain trials.

Within the model just proposed, the effect of catch trials on IHTT, as suggested in Table 1, may be located in three places. First, it is possible that IHT is best captured with the model by a shift of $R_{0}$. This would indicate that the transfer only comes into play after the processes described by the model have ended-that is, after the detection criterion of the directly stimulated hemisphere has been exceeded. A second possibility is that IHT is described best by a depressed slope of the linear function. This would mean that transmission across the commissures somehow decreases the rate with which information is accumulated (e.g., because of stimulus degradation). Finally, IHT might be captured with the model by a higher detection criterion, suggesting two possible origins: either (1) more information must be provided before a response is initiated on the basis of indirect information than on the basis of direct informa- 


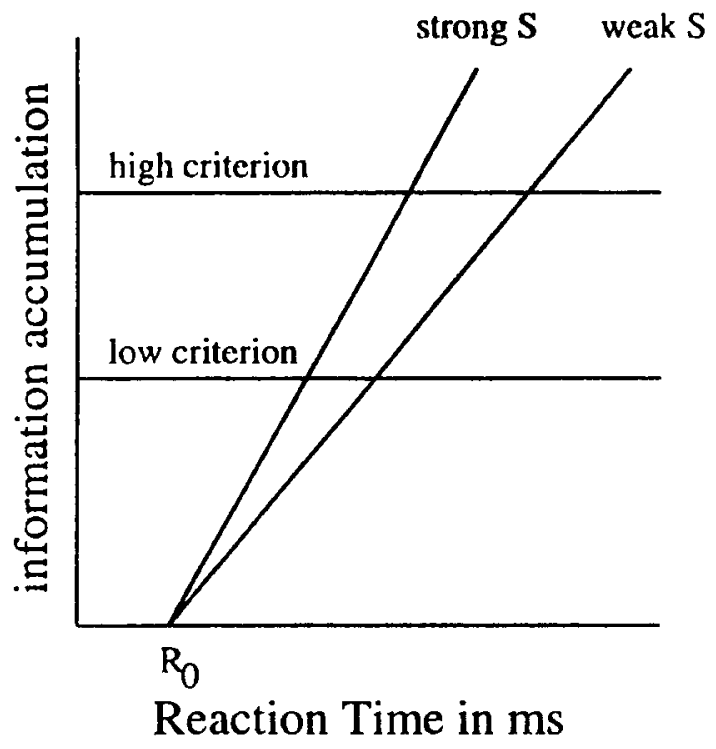

Figure 1. Graphical outline of the variable criterion model. Information accumulation curves for two stimuli (a weak and a strong one) and two different detection criteria (high and low). The expected reaction time in a condition (abscissa) is indicated by the intersection of the lines representing the information accumulation and the detection criterion.

tion (i.e., a threshold difference), or (2) the trajectory to be traversed is longer in the crossed condition than in the uncrossed condition (but without loss of the rate of information buildup). The model does not allow us to distinguish between the latter two alternatives (see the General Discussion). ${ }^{2}$

To investigate which aspect of the model varies between crossed and uncrossed responses, two experiments were run in which an orthogonal variation of stimulus intensity (four levels, within subjects) and percentage of catch trials (three levels including an SRT condition without catch trials, between subjects) was achieved. Experiment 1 deals with unimanual reactions; Experiment 2, with vocal reactions. Previous studies (Clarke \& Zaidel, 1989; Milner \& Lines, 1982, Experiment 1; Tassinari, Morelli, \& Berlucchi, 1983) have failed to find an effect of stimulus intensity on IHTT, so that the slope of the linear function may be the least likely component of the model to vary. As Table 1 suggests, the same need not be true for the criterion level, because the manipulation of the number of catch trials appears to influence IHTT.

\section{EXPERIMENT 1}

\section{Method}

\section{Subjects}

Thirty-six right-handed, male undergraduate students participated on a voluntary basis. Handedness was assessed with a translation of the Oldfield (1971) questionnaire. All subjects were naive with respect to the research question and had normal or correctedto-normal vision.

\section{Procedure}

Stimuli were presented with an IBM AT microcomputer. The computer made use of a Hercules monochrome graphics card with a resolution of $720 \times 348$ pixels and was connected to a black-andwhite LASER monitor, which measured $255 \times 194 \mathrm{~mm}$. Screen luminance, which was controlled with a luminance meter (Minolta NT-1), was adjusted to $36 \mathrm{~cd} / \mathrm{m}^{2}$. Responses were measured with an external response button connected to the parallel port of the microcomputer. Stimulus presentation and response timing were measured to the nearest millisecond with the software routines of Brysbaert and colleagues (Bovens \& Brysbaert, 1990; Brysbaert, 1990; Brysbaert, Bovens, d'Ydewalle, \& Van Calster, 1989).

The stimuli were light flashes presented $75 \mathrm{~mm}$ to the left or right of a fixation mark that consisted of two short vertical lines of 3 pixels separated by a gap of 9 pixels. The subjects were asked to fixate the gap. They sat at a distance of about $60 \mathrm{~cm}$ from the screen (there were no head restraints). At a distance of $57 \mathrm{~cm}$, a stimulus magnitude of $10 \mathrm{~mm}$ coincided with a visual angle of $1^{\circ}$. Stimulus intensity was manipulated by varying the number of pixels of the light flash: The faintest stimulus consisted of a single pixel, followed respectively by rectangular light flashes of $2 \times 2$, $4 \times 4$, and $8 \times 8$ pixels. The experiment was carried out in almost complete darkness; there was only a faint light underneath the table on which the computer was placed. This was done because in pilot studies the subjects had complained of dizziness when they had been sitting in total darkness for a long time.

A trial started with a foreperiod of $1,000 \mathrm{msec}$, followed by a blink of the fixation marker (i.e., it disappeared for $40 \mathrm{msec}$ and then reappeared). Pilot studies had indicated that this procedure would draw the subject's attention (and gaze direction) to the fixation location more than, for instance, an auditory warning signal would. After a second random foreperiod of 400, 450, 500, 550 , or $600 \mathrm{msec}$, the light flash was presented for $20 \mathrm{msec}$ randomly to the left or right of the fixation location. The subject's task was to press the response button as fast as possible. The response button was placed on the midline, and the subject had to react unimanually with the index finger. The hand with which the subject reacted was counterbalanced in an ABBA form; the hand with which he started was also counterbalanced. RTs on stimulus trials shorter than $100 \mathrm{msec}$ or longer than $1,000 \mathrm{msec}$ led to a new presentation of the stimulus at a random place later in the series. The fixation stimulus remained visible throughout the whole experiment.

There were three between-subjects conditions, with different numbers of catch trials. In the first condition, no catch trials were present in a series of 200 trials; the subject had to react on each trial. In the second condition, 20 catch trials were dispersed in a series of 200 stimulus trials, which made a total of 220 trials. During the catch trials, no stimulus was presented and the subject had to withhold the response. Finally, in the third condition, a stimulus series consisted of a random presentation of 120 stimulus trials and 100 catch trials (for randomization procedures, see Brysbaert, 1991). The subjects were explicitly instructed to refrain from response on the catch trials, in order to avoid false alarms. On these trials, the computer waited for $1,000 \mathrm{msec}$, after which the next trial started. At the end of a series, the subjects received feedback about their mean response latency, their standard deviation, the number of RTs shorter than $100 \mathrm{msec}$ or longer than $1,000 \mathrm{msec}$, and eventually the number of false alarms made. The subjects in the first two conditions completed 10 series of 200 stimulus trials; subjects in the third condition finished 20 series of 120 stimulus trials. This made a total of 125 observations per subject in Conditions 1 and 2, and 150 observations in Condition 3 on each of the $2 \times 4 \times 2$ (visual half-field, or VHF $\times$ intensity $\times$ hand) $=16$ within-subjects conditions. There were more observations in the condition with many catch trials, because it was to be expected that this condition, owing to its longer reaction latencies, 
Table 2

Reaction Times from Experiment 1 (Unimanual Responses), as a Function of Visual Half-Field (LVF, RVF), Stimulus Intensity, Responding Hand, and Percentage of Catch Trials

\begin{tabular}{|c|c|c|c|c|c|c|c|c|c|}
\hline \multirow{3}{*}{$\begin{array}{c}\text { Percentage } \\
\text { Catch Trials }\end{array}$} & \multirow[b]{3}{*}{ Hand } & \multicolumn{8}{|c|}{ Stimulus Intensity } \\
\hline & & \multicolumn{4}{|c|}{ LVF } & \multicolumn{4}{|c|}{ RVF } \\
\hline & & 1 & 2 & 3 & 4 & 1 & 2 & 3 & 4 \\
\hline $0 / 200$ & $\begin{array}{l}\text { R } \\
\mathrm{L}\end{array}$ & $\begin{array}{l}269.04 \\
266.40\end{array}$ & $\begin{array}{l}250.97 \\
247.90\end{array}$ & $\begin{array}{l}239.51 \\
233.71\end{array}$ & $\begin{array}{l}231.95 \\
228.02\end{array}$ & $\begin{array}{l}263.70 \\
262.34\end{array}$ & $\begin{array}{l}244.66 \\
246.99\end{array}$ & $\begin{array}{l}234.98 \\
234.16\end{array}$ & $\begin{array}{l}227.14 \\
227.81\end{array}$ \\
\hline $20 / 220$ & $\begin{array}{l}\mathbf{R} \\
\mathbf{L}\end{array}$ & $\begin{array}{l}304.75 \\
309.60\end{array}$ & $\begin{array}{l}285.83 \\
286.01\end{array}$ & $\begin{array}{l}270.34 \\
274.40\end{array}$ & $\begin{array}{l}260.25 \\
261.19\end{array}$ & $\begin{array}{l}301.41 \\
315.00\end{array}$ & $\begin{array}{l}279.36 \\
285.89\end{array}$ & $\begin{array}{l}266.73 \\
274.63\end{array}$ & $\begin{array}{l}255.28 \\
264.97\end{array}$ \\
\hline $100 / 220$ & $\begin{array}{l}\mathbf{R} \\
\mathrm{L}\end{array}$ & $\begin{array}{l}333.57 \\
331.72\end{array}$ & $\begin{array}{l}310.50 \\
309.63\end{array}$ & $\begin{array}{l}297.21 \\
292.51\end{array}$ & $\begin{array}{l}285.78 \\
283.82\end{array}$ & $\begin{array}{l}331.33 \\
330.31\end{array}$ & $\begin{array}{l}307.64 \\
307.22\end{array}$ & $\begin{array}{l}293.24 \\
294.99\end{array}$ & $\begin{array}{l}281.86 \\
285.75\end{array}$ \\
\hline
\end{tabular}

Note-R, right; L, left.

would lead to larger standard deviations (RT distributions are characterized by a positive correlation between mean and standard deviation). In the beginning of the experiment, all subjects received a series of 20 practice trials to explain the nature of the task. In addition, all series started with 20 practice trials with the characteristics of the test trials (e.g., foreperiods, proportion of catch trials). The results of these practice trials were not stored.There were 12 subjects per between-subjects condition.

\section{Results}

The percentage of stimuli that had to be presented twice because of reaction times that were too short or too long amounted to $6 \%$ in the condition with no catch trials, to $2 \%$ in the condition of $20 / 220$ catch trials, and to $3 \%$ in the condition of $100 / 220$ catch trials. The higher percentage in the condition without catch trials was due to anticipatory reactions (i.e., the tendency to react before the stimulus was presented; remember that the random foreperiod ranged from 400 to $600 \mathrm{msec}$, so that there was an uncertainty as to when the stimulus would appear; see Method section). Percentages of false alarms amounted to $5 \%$ in the $20 / 220$ catch trials and $2 \%$ in the $100 / 220$ catch trials. The number of anticipatory reactions and the number of false alarms are a first indication that our manipulation of the detection criterion was successful.

RTs per condition are given in Table 2. Mean RTs were corrected for observations that after logarithmic transformation were larger than the mean plus three times the standard deviation or shorter than the mean minus three times the standard deviation. The percentage of outliers amounted to some $2 \%$ in all conditions; there were no significant differences between the different conditions. A $3 \times 4 \times 2 \times 2$ analysis of variance (ANOVA) with the variables number of catch trials (between subjects), stimulus intensity, VHF, and hand (within subjects) gave the following significant main effects and interactions: main effects of number of catch trials $[F(2,33)=11.8$, $p<.01]$ and stimulus intensity $[F(3,99)=1,114.2, p<$ $.01]$, an interaction between number of catch trials and stimulus intensity $[F(6,99)=6.9, p<.01]$, and a sig-
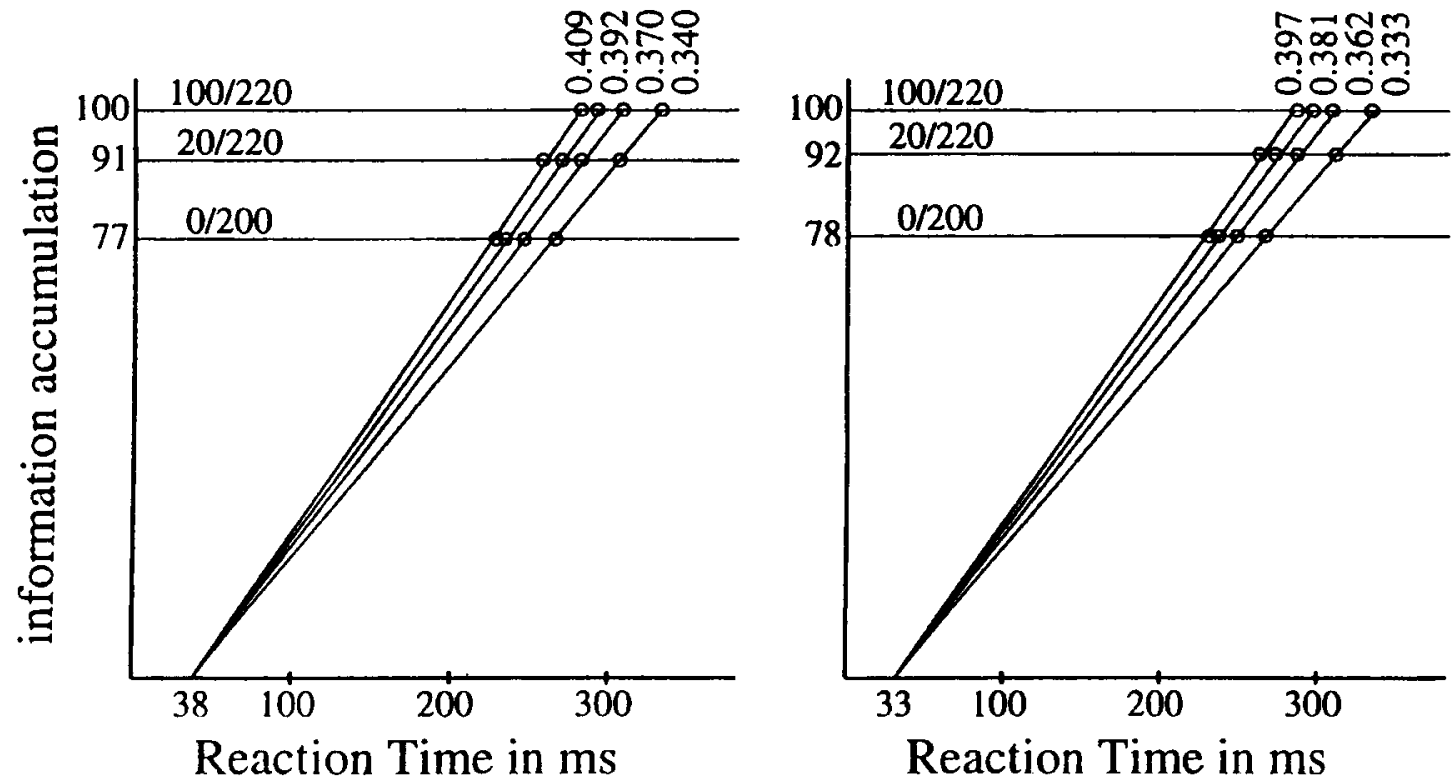

Figure 2. Variable criterion analysis of Experiment 1: four information accumulation curves corresponding to the different stimulus intensities, and three detection criteria according to the percentage of catch trials. Expected values indicated by the intersections of the lines; actual values, by the circles. Left panel, uncrossed conditions; right panel, crossed conditions. 
nificant interaction between hand and $\operatorname{VHF}[F(1,33)=$ $23.4, p<.01]$. Neither the interaction of hand $\times$ VHF $\times$ number of catch trials nor that of hand $\times$ VHF $\times$ stimulus intensity reached significance $(F<1.2)$.

The significant main effects of number of catch trials and stimulus intensity, and their interaction, needed to be present if we wanted to apply the variable criterion model to our results (see the introduction). The significant interaction between VHF and the hand with which the subject responded was expected as well. Of more interest, however, was the absence of a difference in IHTT due to stimulus brightness and number of catch trials. Irrespective of the condition, the estimate equalled about $2.4 \mathrm{msec}$. For stimulus intensity, this was in line with previous studies (Clarke \& Zaidel, 1989; Milner \& Lines, 1982); with respect to the percentage of catch trials, the results contradicted the pattern of data suggested by Table 1 (Bashore, 1981).

The analysis according to the variable criterion model consisted of the following steps (Grice, 1968; Luce, 1986, p. 86): (1) Because it could be assumed that subjects utilized a single criterion for the detection of the different stimulus intensities that were presented in a random order, the average RT was calculated for each of the three levels of catch trials, (2) the slowest average was set arbitrarily to a criterion level of 100 units of information buildup, (3) the magnitude of $R_{0}$ was chosen and subtracted from the average RT coinciding with the different detection criteria, (4) the values of the remaining criterion levels were calculated in units of information build-up by taking the ratio of the time corresponding to these levels relative to the time of the slowest condition, after subtraction of $R_{0},(5)$ the slopes of the information accumulation for the different stimulus intensities were determined with the use of the equation $A_{i}=\mathrm{RT}_{\mathrm{i}} / C$, in which $A_{i}$ is the slope for the $i$ th stimulus intensity, $\mathrm{RT}_{i}$ is the mean RT for the $i$ th stimulus intensity after subtraction of $R_{0}$, and $C$ is the mean of the different criterion levels expressed in units of information buildup, (6) the fit between the observed and the predicted data was calculated, and (7) the value of $R_{0}$ was determined that led to the best fit.

Figure 2 displays the results of the analysis for the uncrossed (left panel) and crossed responses (right panel) separately. ${ }^{3} \mathrm{~A}$ look at $R_{0}$ indicates that the difference between the crossed and the uncrossed conditions is unlikely to be due to a difference in processes unrelated to sensory processing or criterion setting. If anything can be said about $R_{0}$, the results tend to be opposite to the expectations: $R_{0}$ is larger for uncrossed responses $\left(R_{0}=\right.$ $38 \mathrm{msec})$ than for crossed responses $\left(R_{0}=33 \mathrm{msec}\right)$. In principle, the difference could be attributed to a different slope of the information accumulation curves, because the slopes tend to be slightly higher in the uncrossed than in the crossed conditions. However, the slopes are partly determined by the value of $R_{0}$ (if everything else remains constant, the slope increases as $R_{0}$ increases). If $R_{0}$ is fixed at $33 \mathrm{msec}$ for both crossed and

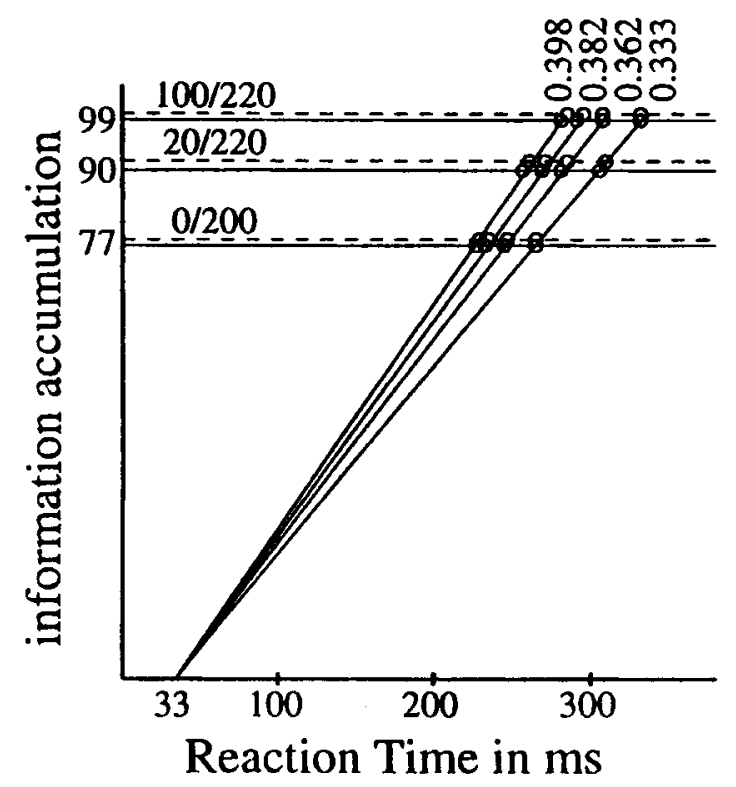

Figure 3. Variable criterion analysis of Experiment 1: combined data for crossed and uncrossed conditions. Numbers on the ordinate indicate the criterion values of the uncrossed conditions (indicated by solid lines). Those of the crossed conditions (dashed lines) are one or two units higher.

uncrossed responses, none of the slopes differs more than .004 . Moreover, if the difference between crossed and uncrossed responses is due to a difference in slope, one would expect IHTT to be larger in conditions with a high criterion level than in conditions with a low criterion level. This is not the case: IHTT equals on the average $2.1 \mathrm{msec}$ in the $0 / 200$ catch trial condition, $3.5 \mathrm{msec}$ in the $20 / 220$ condition, and $1.8 \mathrm{msec}$ in the $100 / 220$ condition. ${ }^{4}$ So, it seems that the difference between crossed and uncrossed responses may best be interpreted as a change in detection criterion. Figure 3 gives the results of the overall analysis of crossed and uncrossed conditions based on that assumption. The fit of the model can be expressed by the ratio of variance explained by the model relative to the total variance around the mean; this fit amounts to $R^{2}=.9990$.

\section{Discussion}

Experiment 1 dealt with the combined effect of stimulus intensity and percentage of catch trials on unimanual IHTT estimates. Previous results (Clarke \& Zaidel, 1989; Milner \& Lines, 1982, Experiment 1) had suggested that stimulus intensity would have little or no effect, but that the percentage of catch trials might be a substantial mediator (Table 1; Bashore, 1981). The empirical results, however, failed to provide evidence for the latter expectation: IHTT estimates were unaffected both by manipulation of the stimulus intensity and by the percentage of catch trials. In addition, Bashore's (1981) thesis that signal detection leads to unstable estimates of IHTT was not confirmed. 
A closer look at the studies reported in Bashore (1981) suggests a possible reason for the unreliable estimates of IHTT found in previous SD studies. What Bashore overlooked in his review was the number of observations on which the estimates were based (see Table 1 of the present manuscript). In SRT tasks, the mean number of stimuli presented to a subject amounted to 1,354 (range, $480-4,000$ ); in SD experiments, it was only 110 (range, 30-200; two experiments gave too little information for the number of observations per subject to be calculated). ${ }^{5}$ Given the large variability of reaction times, the diverging IHTT estimates found in SD experiments may very well have been due to this simple methodological flaw. At least the present study suggests that if a reasonable number of stimuli are presented, the data from conditions with catch trials can be as clear as those from conditions without blank trials; both paradigms return IHTT estimates of 2-3.5 msec. This is within the normal range (Marzi et al., 1991), though somewhat at the low end. It may be interesting to note that Berlucchi, Heron, Rizzolatti, and Umiltà (1977, Experiment 2) obtained about the same estimate in a go/no-go task with a sufficient number of observations per subject (i.e., 1,280), in which the subject had to press on a switch when the stimulus was presented on the same (or the opposite) side of the switch. The estimates were comparable, whether the subject had to press the switches with the hands in the coinciding hemispace or with the hands crossed, so that the effect could not be due to spatial compatibility.

The data from the present experiment were analyzed further with the variable criterion model of Grice (1968), to find out what aspect of the model was most likely to be influenced by the difference between crossed and uncrossed responses. With all the evidence taken together, the findings were most in line with an increased detection criterion for crossed responses relative to uncrossed responses; this implies (1) that more information needs to be accumulated before a response is initiated on the basis of signals sent by the contralateral cerebral hemisphere than on the basis of signals sent by the ipsilateral hemisphere, or (2) that the trajectory that must be traversed is longer in the crossed than in the uncrossed conditions (see the introduction). The rate of information accumulation does not seem to differ between direct and indirect input (Figure 3).

If the idea is correct that IHTT results in an augmented detection criterion, it follows that IHTT must be larger for weak stimuli than for strong stimuli (see the interaction in Figure 1 and Figure 3). However, Figure 3 also indicates that the difference will be virtually unobservable in normal subjects, because the detection criteria for contralateral and ipsilateral stimuli flank one another. Only if the disparity grows larger is it possible to find a reliable interaction between stimulus intensity and crossed/uncrossed responses. This leads to the question whether the interaction would appear in data from splitbrain patients. For in these subjects, it has been demonstrated that the difference between crossed and uncrossed responses is in the order of a few tens of milliseconds

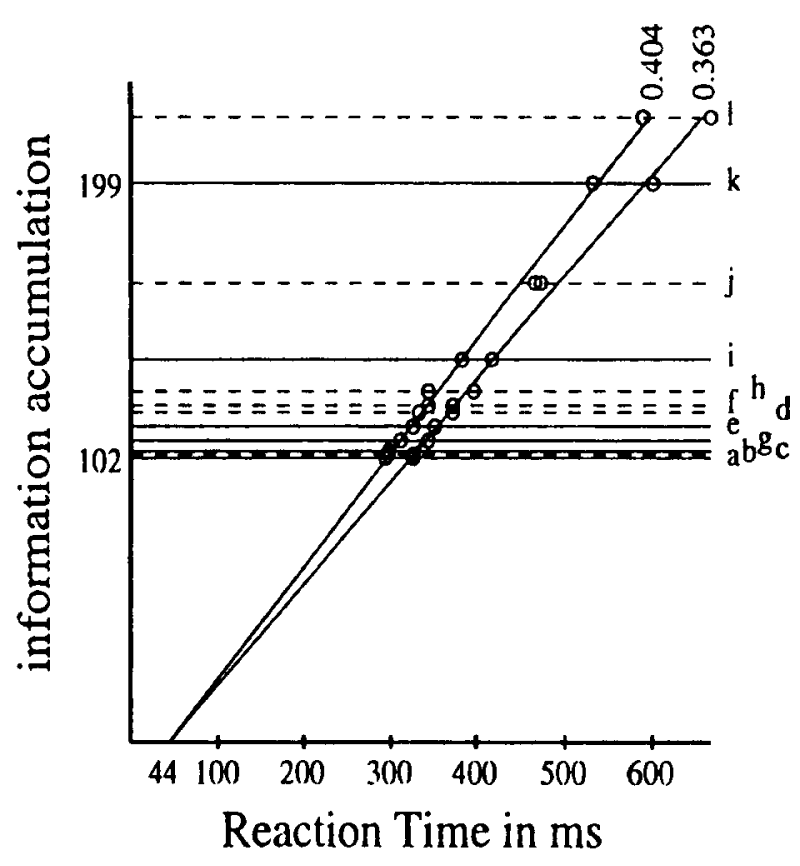

Figure 4. Variable criterion analysis of Clarke and Zaidel (1989, Experiment 1). Two different stimulus intensities and six (groups of) subjects. Solid lines represent the uncrossed conditions (a, normals; c, L.B.; e, M.M.; g, N.G.; i, R.Y.; k, A.A.) and dashed lines the crossed conditions (b, normals; d, L.B.; f, M.M.; h, N.G.; j, R.Y.; L, A.A.).

rather than a few milliseconds (e.g., Clarke \& Zaidel, 1989; Jeeves, 1969). So if the model is correct, these patients should show larger IHTTs for weak stimuli than for strong stimuli, which has indeed been reported (Clarke \& Zaidel, 1989; Milner, Jeeves, Silver, Lines, \& Wilson, 1985).

To test the viability of the model for both normal subjects and split-brain patients more directly, all data reported by Clarke and Zaidel (1989, Experiment 1$)^{6}$ were entered in an analysis like the one described in Figure 3. These data consisted of unimanual reactions from 40 normal subjects, 4 commissurotomized patients, and a boy with callosal agenesis (M.M.), who reacted to two different stimulus intensities and made a total of 800 responses. Figure 4 displays the outcome of the analysis.

The parameters of the model agreed quite well with those obtained in Experiment 1. $R_{0}$ amounted to $44 \mathrm{msec}$ (it was $33 \mathrm{msec}$ in our study), the slopes were within the same range if the same criterion level was used as a reference $(0.35-0.40$ information units per time unit), and the overall fit had a similar magnitude $\left(R^{2}=.9933\right)$. Only the data of the crossed condition from Subject R.Y. (line j) did not correspond to the predictions. For comparison, a second model was applied, which assumed an effect of interhemispheric transfer on the information accumulation rather than on the detection criterion. This second model was less successful: Its fit was lower $\left(R^{2}=\right.$ .9870 ), $R_{0}$ was considerably augmented (i.e., $134 \mathrm{msec}$ ), and the slopes were substantially higher (i.e., between 0.51 and 0.68 information units per time unit). The rel- 
atively low fit of the second model is further evidence that the difference between crossed and uncrossed conditions is represented best by a change in detection criterion, at least under the constraints of the variable criterion theory. It may be noteworthy that a similar analysis of eccentricity data (Clarke \& Zaidel, 1989, Experiment 2) did not return the same straightforward results (e.g., $R_{0}=237 \mathrm{msec}$ for the model with different detection criteria), so that the applicability of the model may be limited to stimulus intensity manipulations.

The implications of the preceding findings for the nature of IHT will be discussed at greater length in the General Discussion, after examination of the results of Experiment 2 (vocal reactions). In the literature, different anatomical pathways have been suggested for manual and verbal measures of IHT, so that it may be interesting to compare the outcome of both dependent variables within the framework of the variable criterion model.

\section{EXPERIMENT 2}

In Experiment 1, the distinction drawn by Bashore (1981) between SRT and SD experiments with regard to the assessment of IHTT with unimanual reactions to lateralized light flashes did not occur when the same (large) number of observations was completed in all conditions. This raises the question of whether the same finding would hold for verbal estimates of IHTT, for another paradigm that has been proposed to measure IHTT consists not of having the subjects press a button when a light flash is presented, but of having them say a word as soon as a light is flashed in one or the other VHF (e.g., Milner \& Lines, 1982, Experiment 2). There is good evidence (e.g., Bradshaw, 1989; Bradshaw \& Nettleton, 1983 ; Bryden, 1982) that in $90 \%-95 \%$ of right-handers, speech output is lateralized in the left cerebral hemisphere. So the difference between the RT for stimuli in the LVF and the RT for stimuli in the RVF may yield another estimate of IHTT, because vocal reactions to stimuli in the LVF require IHT, whereas reactions to stimuli in the RVF do not.

Table 3

Reaction Time (RT) Studies of Interhemispheric Transmission Time (IHTT) Based on Verbal Reactions to Lateralized Light Flashes; Distinction Made Between Simple RT Studies and Signal Detection Studies

\begin{tabular}{lccccc}
\hline \multicolumn{1}{c}{ Source } & $N_{\text {subj }}$ & $N_{\text {obs } / \text { subj }}$ & $\mathrm{RT}_{\text {LVF }}$ & $\mathrm{RT}_{\mathrm{RVF}}$ & IHTT (msec) \\
\hline \multicolumn{5}{c}{ Simple RT } \\
Geffen et al., 1971 & 10R & 160 & $380.0^{\mathrm{a}}$ & 380.7 & -0.7 \\
Tassinari et al., 1983 & $8 \mathrm{R}$ & $640^{\mathrm{b}}$ & 267.4 & 267.1 & 0.3 \\
Sergent \& Myers, 1985 & $1 \mathrm{R}$ & 100 & 250.9 & 255.5 & -4.9 \\
St. John et al., 1987 & $12 \mathrm{R}$ & 5,760 & 304.8 & 297.4 & $7.4^{*}$ \\
\multicolumn{5}{c}{ Signal Detection } \\
Kleinman et al., 1976 & 33R & $30 \mathrm{~S} / 15 \mathrm{C}^{\mathrm{c}}$ & 400.0 & 380.0 & $20.0^{*}$ \\
Swanson et al., 1978 & $12 ?$ & $?$ & $?$ & $?$ & $27.0 ?$
\end{tabular}

${ }^{*} p<.05$. ${ }^{a}$ Values not given in the original article, but communicated by Gina Geffen to the author on January 25, 1993 . "Four different responses in different conditions: "si," "no," "sette," and "otto." "S, stimulus trials; $\mathrm{C}$, catch trials.
Probably because of the scarcity of studies that made use of verbal RTs, Bashore (1981) did not draw the distinction between SRT and other, more complex designs (e.g., SD, go/no-go, and choice reaction) in his analysis. He simply listed four references one under the other: none of them made use of SRT, only one (Kleinman, Carron, Cloninger, \& Halvachs, 1976) worked with SD, and the rest consisted of more complicated go/no-go tasks (i.e., reacting to a certain stimulus and not reacting to another one). Therefore, Bashore's conclusion that verbal reactions did not lead to consistent measures of IHTT may have been unwarranted. Table 3 gives a more balanced review of IHTT estimates based on verbal responses, with four SRT studies and two SD studies. In this table, the same pattern of results seems to emerge as from Table 1: small (but this time inconsistent) IHTTs for SRT tasks, and larger IHTTs for SD experiments. However, some precaution is needed, because (1) the SD references are the same as those which gave rather elevated IHTT estimates in Table 1, and (2) only two of the studies (St. John, Shields, Krahn, \& Timney, 1987; Tassinari et al., 1983 ) administered more than 200 stimuli per subject.

The absence of an LVF-RVF difference for vocal responses in their study, together with the presence of a crossed-uncrossed difference for manual responses made by the same subjects, made Tassinari et al. (1983, p. 77) conclude that: "simple verbal RT is unsuitable for measuring interhemispheric transmission time." They explained this rather counterintuitive statement by assuming that although the organization of speech is lateralized to the left hemisphere, the initiation of verbal responses can be controlled by both hemispheres through subcortical (i.e., diencephalic and mesencephalic) centers. Support for Tassinari et al.'s explanation came from a study by Sergent and Myers (1985), who obtained unimanual and vocal IHTT measures from two commissurotomized patients. While the unimanual responses gave rise to the expected, highly elevated IHTT in the order of 30-50 msec (see above), the verbal responses only led to insignificant LVF-RVF differences of $4.8 \mathrm{msec}$ (Subject L.B.) and $2.9 \mathrm{msec}$ (N.G.). It should be noted, however, that Tassinari et al.'s explanation is not in line with the significant LVF-RVF difference found by St. John et al. (1987; see Table 3 in the present paper).

The picture of IHTT estimates based on verbal responses is further complicated by two studies (Lines \& Milner, 1983; Milner \& Lines, 1982, Experiment 2) in which the subjects were screened before taking part in the experiment. Subjects who failed to show an overall RVF advantage were excluded from the analysis. In Milner and Lines (1982), this resulted in the exclusion of 12 of the 24 subjects; in Lines and Milner (1983) only 4 of the 28 subjects needed to be excluded. For the present discussion, the Milner and Lines (1982) paper is the most interesting one, because in this study the authors manipulated the stimulus intensity and reported a larger LVF-RVF difference for faint stimuli than for bright stimuli: Over four increasing stimulus intensities, the IHTT estimate decreased from 12 to $4 \mathrm{msec}$. This led Milner 
and Lines to conclude that vocal responses, unlike unimanual reactions, required the transmission of a visual stimulus. Tassinari et al. (1983) criticized Milner and Lines's interpretation because of the selection of subjects.

To clarify the diverging findings on verbal IHTT estimates, it seems that a few guidelines need to be advanced. First, the distinction between types of tasks successfully introduced in the literature on unimanual estimates should be accredited in verbal tasks as well. Therefore, in this paper only SRT and SD experiments will be discussed (see Table 3). Both paradigms require one response to one stimulus and differ only in terms of the presence of catch trials with no stimulus and no answer. Second, as has been argued in the discussion section of Experiment 1, it is dangerous to base conclusions on less than 500 observations per subject. The differences we are looking for are so small that the designs must have enough power to make an estimate of a few milliseconds reliable. Third, it must be acknowledged that studies with verbal responses have one major drawback that studies with manual responses do not havenamely, the fact that there is a confounding between the VHF and direct-indirect input. Estimates based on manual reactions are obtained by an orthogonal variation of the responding hand and the VHF stimulated, which is not possible for verbal responses. So estimates based on verbal responses have the same status as IHTT estimates based on LVF-RVF differences for the right hand only. All else remaining constant, it can be expected that these measures, by their very nature, are more variable. This has to do with the fact that RT differences between stimuli presented to the left or to the right of fixation are not uniquely determined by cerebral asymmetry but also by a "characteristic perceptual asymmetry" (see, e.g., Kim \& Levine, 1991). People differ in the amount of attention that they allocate to the left or the right VHF, and/or in the speed with which information is transferred from the hemiretinas through the cortex. This has consequences even for the very simple tasks discussed here, as can easily be checked from Experiment 1 . If the data are rearranged according to the VHF that leads to faster overall processing, a mean VHF superiority of $6 \mathrm{msec}$ is obtained. In agreement with the variable criterion hypothesis, the difference is larger for faint stimuli than for bright stimuli (i.e., 7.7, 6.8, 5.4, and $4.8 \mathrm{msec}$ for the increasing intensities). A similar VHF $\times$ stimulus intensity interaction has been reported after explicit attention manipulation (Hawkins, Shafto, \& Richardson, 1988), so that the results of Milner and Lines (1982) reported above presumably say more about the effects of attention allocation and/or processing speed than about IHT. An average VHF superiority of $6 \mathrm{msec}$ to either side of the fixation location implies a dramatic increase in the variability of the LVF-RVF difference, which can only be surmounted by a sufficiently large sample of subjects.

If we then look at the studies that have successfully met the above three criteria (Table 3), we see that only the studies of St. John et al. (1987) and, to a lesser extent, Tassinari et al. (1983) pass the test. Even then, ran- dom fluctuations in the characteristic perceptual asymmetry of the subjects may very well have accounted for the lack of an LVF-RVF difference in Tassinari et al., and for the rather pronounced difference in St. John et al. The experiment below gives data from 72 subjects who finished 1,200 stimulus trials each.

\section{Method}

\section{Subjects}

Seventy-two right-handed male undergraduate students participated on a voluntary basis. They were naive with respect to the purpose of the Experiment and had normal or corrected-to-normal vision. None of the subjects had participated in the previous experiment. The handedness of the subjects was assessed with a translation of the Oldfield (1971) questionnaire.

\section{Procedure}

The equipment was the same as in Experiment 1. The only difference was that in the present experiment, the response button was replaced by a microphone connected to the game port of the microcomputer. The subjects had to say "ja" (i.e., the Dutch word for "yes") as soon as they saw a light flash. Because the subjects no longer reacted manually, the number of within-subjects conditions could be halved (there was no need to change the hand with which the subject responded). However, because vocal estimates of IHTT are expected to be more variable than manual estimates (see above), the number of subjects was double that in Experiment 1 . In the conditions with no catch trials and with $20 / 220$ catch trials, six series of 200 stimulus trials were presented; in the condition with $100 / 220$ catch trials, 10 series of 120 stimulus trials were administered. This made a total of 150 observations per subject on each of the $2 \times 4$ (VHF $\times$ intensity) $=8$ within-subjects conditions. The stimulus presentation, including that on the practice trials, was the same as in Experiment 1. There were 24 subjects per between-subjects condition.

\section{Results}

The percentage of stimuli that had to be presented twice because RTs were shorter than $100 \mathrm{msec}$ or longer than $1,000 \mathrm{msec}$ amounted to $6 \%$ in the condition with no catch trials, $3 \%$ in the condition with $20 / 220$ catch trials, and $2 \%$ in the condition with $100 / 220$ catch trials. The higher percentage in the condition without catch trials was again due to anticipatory reactions. The percentage of false alarms amounted to $8 \%$ in the condition with $20 / 220$ catch trials, and to $4 \%$ in the condition with $100 / 220$ catch trials. The percentages of false alarms were slightly overestimated in this experiment because on a number of occasions noise caused a signal in the

Table 4

Reaction Times from Experiment 2 (Verbal Responses), as a Function of Visual Half-Field (LVF, RVF), Stimulus Intensity, Responding Hand, and Percentage of Catch Trials

\begin{tabular}{llcccc}
\hline \multirow{2}{*}{$\begin{array}{c}\text { Percentage } \\
\text { Catch Trials }\end{array}$} & VHF & 1 & \multicolumn{4}{c}{ Stimulus Intensity } \\
\cline { 2 - 6 } $0 / 200$ & LVF & 332.68 & 317.25 & 306.01 & 296.99 \\
& RVF & 330.16 & 312.69 & 302.69 & 293.57 \\
$20 / 220$ & LVF & 355.12 & 335.27 & 323.13 & 312.52 \\
& RVF & 352.64 & 330.79 & 318.40 & 309.14 \\
$100 / 220$ & LVF & 416.75 & 391.78 & 376.74 & 365.55 \\
& RVF & 417.08 & 392.59 & 376.65 & 365.19 \\
\hline
\end{tabular}


catch trials, which was erroneously registered as a false alarm by the system.

Average RTs per condition can be found in Table 4 . Outlier detection occurred as in Experiment 1 and yielded the same percentage of observations (i.e., around $2 \%$ ) that after logarithmic transformation were larger than the mean plus three times the standard deviation or smaller than the mean minus three times the standard deviation. A $3 \times 4 \times 2$ ANOVA with the variables number of catch trials (between subjects), stimulus intensity, and VHF (within subjects) gave the following significant main effects and interactions: There were main effects of number of catch trials $[F(2,69)=13.1, p<.01]$ and stimulus intensity $[F(3,207)=956.0, p<.01]$, as well as a significant interaction between both $[F(6,207)=$ $10.6, p<.01]$; there was also a significant main effect of VHF $[F(1,69)=7.2, p<.01]$, but no interaction between VHF and number of catch trials $[F(2,69)=2.1$, $p>.10]$, or between VHF and stimulus intensity $(F<1)$.

The main effects of number of catch trials and stimulus intensity, as well as their interaction, replicate the findings of Experiment 1 and show that the variable criterion model is applicable to the data. The main effect of VHF was reliable and amounted to $2.3 \mathrm{msec}$, a value very close to the IHTT estimate obtained in Experiment 1 and smaller than the absolute LVF-RVF difference per subject which on the average amounted, respectively, to $6.4,8.1,5.4$, and $4.8 \mathrm{msec}$ for the increasing stimulus intensities.

Application of the variable criterion model to the LVF and RVF data separately at first sight gave evidence for a shift in $R_{0}(\mathrm{LVF}=128 ; \mathrm{RVF}=107)$. However, because

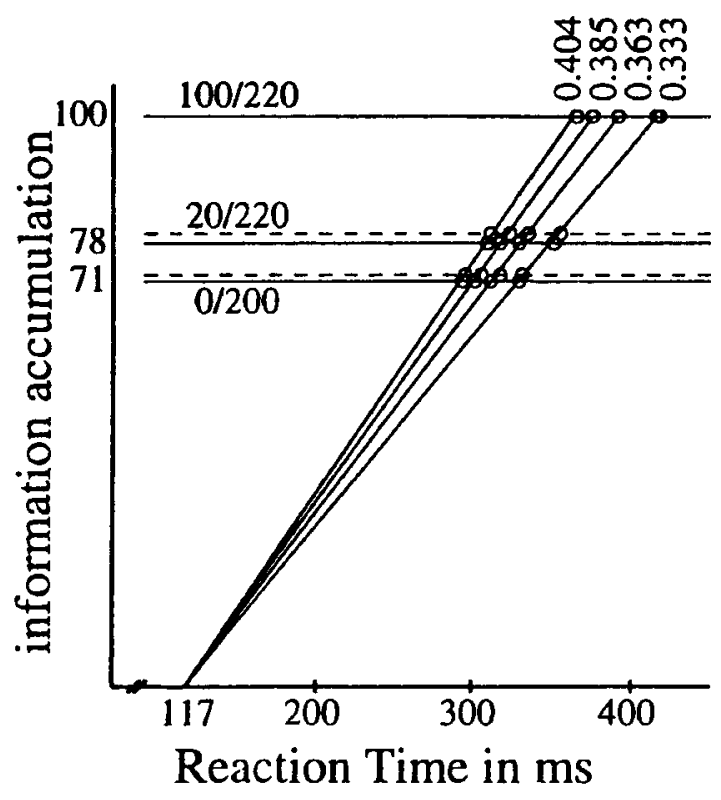

Figure 5. Variable criterion analysis of Experiment 2: combined data for crossed and uncrossed conditions. Numbers on the ordinate indicate the criterion values of the uncrossed conditions (solid lines). Those of the crossed conditions (dashed lines) are slightly higher, except for the 100/220 condition, in which the criteria coincided. the shift was larger than the 2-msec difference obtained in the raw data, and because it led to slopes different from the ones obtained in Experiment 1, we decided to run the model proposed for the combined data of Experiment 1 , in which the only difference between crossed and uncrossed responses was situated in the detection criteria. The result of the analysis is given in Figure 5; the fit of the model amounted to $R^{2}=.9996{ }^{7}$

Comparison of the data displayed in Figure 5 with the data from Experiment 1 (Figure 3) shows that the major difference between the two tasks was situated in $R_{0}$ that is, the time needed to execute the actual movement once the detection criterion had been exceeded. It took about $80 \mathrm{msec}$ longer for a microphone to detect the sound "ja" than for a response button to register a finger press. There were only a few minor changes in the relative position of the detection criteria. The slopes of the information accumulation were virtually identical.

\section{Discussion}

An analysis of the task characteristics inherent in verbal estimates of IHTT indicated that these estimates are likely to be more variable than manual estimates, because they are confounded with attention allocation to the left and right of fixation and/or with processing speed for stimuli presented in the LVF and RVF. This implies that in addition to the large number of observations needed for reliable individual measurements, a substantial sample of the population must be examined to bypass the variability introduced by the characteristic perceptual asymmetry. A review of the literature (Table 3 ) indicated that only two SRT studies and no SD study approached meeting both criteria.

An implementation of the criteria in an actual experiment resulted in the unexpected finding that the results were an exact replica of those obtained with unimanual responses, except for the time needed between the initiation of the response and the actual registration of the answer (i.e., $R_{0}$ ). The information accumulation slopes were virtually the same and the difference between crossed and uncrossed responses was well captured by a small change in the detection criterion. No significant differences in IHTT were found between the SRT and SD conditions.

In sum, there seems to be no need to invoke different models for verbal and manual responses to lateralized light flashes, just as the manual data of normal and commissurotomized subjects could be described within the same framework (Figure 4). This leaves open the question of why split-brain patients exhibit a considerably smaller crossed-uncrossed difference for verbal responses than for manual responses. This question will be dealt with in the general discussion.

\section{GENERAL DISCUSSION}

In 1981, Bashore got order out of the literature on behavioral estimates of interhemispheric transmission time by pointing out that only SRT tasks with manual re- 
sponses yielded consistent measures. Since then, this picture has virtually remained unchallenged and has led to the almost exclusive use of manual SRT experiments for the investigation of IHTT. However, a closer inspection of the studies reported by Bashore indicated that the distinction between different research paradigms coincided with differences in methodological rigor. SRT studies with manual responses happened to be the only studies in which a large number of observations was invariably secured from a considerable number of subjects. Although the other paradigms could be expected to yield more variable RTs, they were tested with fewer trials and/or subjects. When the numbers of trials and subjects were equated in the SRT and SD conditions, the resulting IHTT estimates turned out to be virtually the same. This was true for measurements based on unimanual responses and for measurements based on verbal responses, which suggests that the four paradigms can be used jointly to look for converging patterns of evidence.

The equivalence of the SRT and the SD paradigm need not imply that the same will be true for other, more complex designs such as choice reaction tasks and go/ no-go tasks. The common element of SRT and SD is that subjects give a simple reaction as soon as an unstructured stimulus appears; the stimulus needs no analyzing. There is quite good neurophysiological evidence (e.g., Kaas, 1989) that the visual system consists of two distinct pathways; the parvocellular pathway, which plays a major role in object recognition, and the magnocellular pathway, which mediates rapid responses to stimulus changes-in particular, stimulus movement (but also stimulus onset). Given the short RTs, the Poffenberger task is likely to measure processes of the fast magnocellular pathway, and not of the slower parvocellular pathway. This would be in line with the finding that VHF asymmetries are more pronounced in experiments that require stimulus analysis than in those that do not (e.g., Geffen, Bradshaw, \& Wallace, 1971; Tassinari et al., 1983). It would also imply that the Poffenberger task is not well suited for measuring IHT of pattern information.

Apart from the preceding methodological rectification, Experiments 1 and 2 have also introduced a simple mathematical model to analyze data of Poffenberger experiments in which an orthogonal variation of stimulus and response aspects has been achieved. The model is called the variable criterion model and is due to Grice (1968, note 1). If it is applied to SRT and SD data, a quite interesting picture emerges. First, the introduction of catch trials is described very well by a change of the detection criterion, as can be concluded from the interaction between stimulus intensity and percentage of catch trials in Experiments 1 and 2 . There has been evidence suggesting that the interaction is more difficult to obtain with visual stimuli than with auditory stimuli (Grice, Nullmeyer, \& Schnizlein, 1979; Nissen, 1977), but this does not seem to hold for the manipulation realized here (see also Hawkins et al., 1988). The introduction of catch trials has the effect that more information must be gathered before a response is initiated, as could already be concluded from the percentage of anticipatory reactions and false alarms in the different conditions (see the Results sections). Second, the rate of information accumulation and the criterion setting are identical for manual and verbal responses, which casts doubts on the claim that the two reaction types use different anatomical pathways (Tassinari et al., 1983). Third, the rate of information buildup does not seem to be severed in patients without corpus callosum (be it commissurotomy or agenesis), as can be concluded from the fact that a model with about the same linear slopes can be applied to the findings of Clarke and Zaidel (1989). Fourth, the effect of IHT is best explained in terms of detection criterion setting: The criterion is higher for crossed than for uncrossed responses. This is true both for normals and for acallosals.

Because the detection criterion is determined by the amount of information that needs to be accumulated before a response is initiated, as well as by the length of the trajectory that the information has to go through (see the introduction), three different explanations might be given for the difference between crossed and uncrossed responses. The first explanation, formulated entirely in terms of the amount of information that must be accumulated, says that more signals must be gathered if they come from the contralateral hemisphere than if they come from the ipsilateral hemisphere. A way of conceiving this might be that the stimulus, in addition to triggering the response device, has an effect on the alertness of the hemisphere it is primarily sent to, so that the detection criterion of that hemisphere is slightly lowered. It is well known that warning signals decrease the RT, even if the warning signal is presented simultaneously with the imperative stimulus (e.g., Nissen, 1977, p. 347). The second explanation says that IHTT is due exclusively to the distance that the signals have to traverse before they reach the detection criterion. This is the explanation usually advocated by researchers of IHT (Bashore, 1981; Marzi et al., 1991). The third explanation, finally, treats IHTT as a combination of these two factors. For example, one version might be that the 2- to 4-msec difference in normals and in the verbal data of split-brains is caused by the length of the anatomical pathway, whereas the rest of the difference for manual responses in split-brain patients is due to attentional imbalances. It has been noted before that one of the major symptoms of commissurotomy is the difficulty to switch attention from one side to the other (see, e.g., Gazzaniga, 1987; Reuter-Lorenz \& Fendrich, 1990; Wale \& Geffen, 1989). For some yet-to-be-explained reasons, the switching may be more difficult when the hand has to respond than when the mouth is involved. At least the third explanation would account for the problem that present theories have in explaining why the subcortical pathway that has been invoked for verbal responses in acallosals does not take over the function of the corpus callosum in the Poffenberger task with manual reactions.

The use of the mathematical model may be criticized because its starting point is the overall pattern of results 
and not the different anatomical pathways that may be involved in IHT. Although it is true that the application of a mathematical model entails the risk of biased interpretation, it should be noted that the anatomically oriented research is not amodal either. More specifically, it relies heavily on Sternberg's stage model (Sternberg, 1969), which considers the separate information processing steps as completely autonomous and uninfluenced by one another. So, if the IHTT does not depend on stimulus factors such as intensity and eccentricity, it is concluded that the signal transferred between the brain halves is of a nonperceptual nature. On the basis of that reasoning, IHT has been thought to be perceptual (see, e.g., Milner \& Lines, 1982, Experiment 1), nonperceptual (e.g., Milner \& Lines, 1982, Experiment 2), or nonexisting (e.g., Tassinari et al., 1983) for different tasks and subject populations. And on the basis of the present results, one would have to conclude that the information is neither perceptual nor motor, as both manipulations do not have a significant influence on IHTT. In addition, the validity of the strong anatomical model has recently been put into question, because evidence suggests that the information transfer in the Poffenberger task happens simultaneously at different stages of processing (Bisiacchi et al., in press; Tassinari, Aglioti, Pallini, Berlucchi, \& Rossi, in press). It no longer seems inconceivable that communication between the hemispheres occurs in parallel in different places such as the visual cortex, the premotor cortex, and the subcortical pathway. This agrees with the present mathematical model, which states that what is important in the Poffenberger task is not the particular pathway followed by the signals, but the total amount of information buildup and the level of the detection criterion.

\section{REFERENCES}

Anzola, G. P., Bertoloni, G., Buchtel, H. A., \& Rizzolatti, G. (1977). Spatial compatibility and anatomical factors in simple and choice reaction time. Neuropsychologia, 15, 295-305.

BASHORE, T. R. (1981). Vocal and manual reaction time estimates of interhemispheric transmission time. Psychological Bulletin, 89, 352-368.

Berlucchi, G., Crea, F., DiStefano, M., \& Tassinari, G. (1977). Influence of spatial stimulus-response compatibility on reaction time of ipsilateral and contralateral hand to lateralized light stimuli. Journal of Experimental Psychology: Human Perception \& Performance, 3, 505-517.

Berlucchi, G., Heron, W., Rizzolatti, G., \& Umiltà, C. (1971). Simple reaction times of ipsilateral and contralateral hand to lateralized visual stimuli. Brain, 94, 419-430.

Bisiacchi, P., Marzi, M. A., Nicoletti, R., Carena, G., MucigNAT, C., \& TOMaIUOLo (in press). Left-right asymmetry of callosal transfer in normal human subjects. Behavioural Brain Research.

Bovens, N., \& BRysbaerT, M. (1990). IBM PC/XT/AT and PS/2 Turbo Pascal timing with extended resolution. Behavior Research Methods, Instruments, \& Computers, 22, 332-334.

Bradshaw, J. L. (1989). Hemispheric specialization and psychological function. Chichester, U.K.: Wiley.

BRAdShaW, J. L., \& NetTleton, N. C. (1983). Human cerebral asymmetry. Englewood Cliffs, NJ: Prentice-Hall.

BRYDEN, M. P. (1982). Laterality: Functional asymmetry in the intact brain. New York: Academic Press.
Brysbaert, M. (1990). A warning about millisecond timing in Turbo Pascal. Behavior Research Methods, Instruments, \& Computers, 22 , 344-345.

BRYSBAERT, M. (1991). Algorithms for randomness in the behavioral sciences: A tutorial. Behavior Research Methods, Instruments, \& Computers, 23, 45-60.

Brysbaert, M., Bovens, N., d'Ydewalle, G., \& Van Calster, J. (1989). Turbo Pascal timing routines for the IBM microcomputer family. Behavior Research Methods, Instruments, \& Computers, 21 , 73-83.

Clarke, J. M., \& Zaidel, E. (1989). Simple reaction times to lateralized light flashes: Varieties of interhemispheric communication routes. Brain, 112, 849-870.

DiStefano, M., Morelli, M., Marzi, C. A., \& Berlucchi, G. (1980). Hemispheric control of unilateral and bilateral movements of proximal and distal parts of the arm as inferred from simple reaction time to lateralized light stimuli in man. Experimental Brain Research, 38, 197-204.

DusorR, T. (1983). Isobias curves in some detection tasks. Perception \& Psychophysics, 33, 403-412.

GazZaniga, M. S. (1987). Perceptual and attentional processes following callosal section in humans. Neuropsychologia, 25, 119-133.

GefFen, G., Bradshaw, J. L., \& Wallace, G. (1971). Interhemispheric effects on reaction time to verbal and nonverbal stimuli. Journal of Experimental Psychology, 87, 415-422.

GRICE, G. R. (1968). Stimulus intensity and response evocation. Psychological Review, 75, 359-373.

GRICE, G. R., Nullmeyer, R., \& SCHNizlein, J. (1979). Variable criterion analysis of brightness effects in simple reaction time. Journal of Experimental Psychology: Human Perception \& Performance, 5, 303-314.

Grice, G. R., Nullmeyer, R., \& Spiker, V. A. (1982). Human reaction time: Toward a general theory. Journal of Experimental Psychology: General, 111, 135-153.

HaWkins, H. L., ShafTo, M. G., \& Richardson, K. (1988). Effects of target luminance and cue validity on the latency of visual detection. Perception \& Psychophysics, 44, 484-492.

JEEVES, M. A. (1969). A comparison of interhemispheric transmission times in acallosals and normals. Psychonomic Science, 16, 245-246.

KAAS, J. H. (1989). Changing concepts of visual cortex organization in primates. In J. W. Brown (Ed.), Neuropsychology of visual perception (pp. 3-32). Hillsdale, NJ: Erlbaum.

KIM, H., \& LEviNE, S. C. (1991). Sources of between-subjects variability in perceptual asymmetries: A meta-analytic review. Neuropsychologia, 29, 877-888.

Kleinman, K. M., Carron, R., Cloninger, L., \& Halvachs, P. (1976). A comparison of interhemispheric transmission times as measured by verbal and manual reaction time. International Journal of Neuroscience, 6, 285-288.

LinEs, C. R., \& MiLner, A. D. (1983). Nasotemporal overlap in the human retina investigated by means of simple reaction time to lateralized light flash. Experimental Brain Research, 50, 166-172.

LuCE, R. D. (1986). Response times: Their role in inferring elementary mental organization. New York: Oxford University Press.

Marzi, C. A., BisiacchI, P., \& NicoletTI, R. (1991). Is interhemispheric transfer of visuomotor information asymmetric? Evidence from a meta-analysis. Neuropsychologia, 29, 1163-1177.

MilneR, A. D., JeEves, M. A., Silver, P. H., Lines, C. R., \& WiLSON, J. (1985). Reaction times to lateralized visual stimuli in callosal agenesis: Stimulus and response factors. Neuropsychologia, 23, 323-331.

Milner, A. D., \& Lines, C. R. (1982). Interhemispheric pathways in simple reaction time to lateralized light flash. Neuropsychologia, 20, 171-179.

Moscovitch, M., \& Sмiтh, L. C. (1979). Differences in neural organization between individuals with inverted and noninverted handwriting postures. Science, 205, 710-713.

NisSEN, M. J. (1977). Stimulus intensity and information processing Perception \& Psychophysics, 22, 338-352.

OLDFIELD, R. C. (1971). The assessment and analysis of handedness: The Edinburgh inventory. Neuropsychologia, 9, 97-113. 
Poffenberger, A. T. (1912). Reaction time to retinal stimulation, with special reference to the time lost through nerve centers. Archives of Psychology, 23, 1-73.

Reuter-LoRenZ, P. A., \& FENDRICH, R. (1990). Orienting attention across the vertical meridian: Evidence from callosotomy patients. Journal of Cognitive Neuroscience, 2, 232-238.

Sergent, J., \& MYers, J. J. (1985). Manual, blowing, and verbal simple reactions to lateralized flashes of light in commissurotomized patients. Perception \& Psychophysics, 37, 571-578.

SмITH, F. O. (1938). An experimental study of the reaction time of the cerebral hemispheres in relation to handedness and eyedness. Journal of Experimental Psychology, 22, 75-83.

Sмiтh, L. C., \& Moscovitch, M. (1979). Writing posture, hemispheric control of movement and cerebral dominance in individuals with inverted and noninverted postures during writing. Neuropsychologia, 17, 637-644.

St. John, R., Shields, C., Krahn, P., \& Timney, B. (1987). The reliability of estimates of interhemispheric transmission times derived from unimanual and verbal response latencies. Human Neurobiology, 6, 195-202.

STERNBERG, S. (1969). The discovery of processing stages: Extensions of Donders' method. Acta Psychologica, 30, 276-315.

SWANSON, J., LeDlow, A., \& KinSBouRne, M. (1978). Lateral asymmetries revealed by simple reaction time. In M. Kinsbourne (Ed.), Asymmetrical function of the brain (pp. 274-291). Cambridge: Cambridge University Press.

Tassinari, G., Aglioti, S., Pallini, R., Berlucchi, G., \& Rossi, G. F. (in press). Interhemispheric integration of simple visuomotor responses in patients with partial callosal defects. Behavioural Brain Research.

Tassinari, G., Morelli, M., \& Berlucchi, G. (1983). Interhemispheric transmission of information in manual and verbal reactiontime tasks. Human Neurobiology, 2, 77-85.

WALE, J., \& GEFFEN, G. (1989). Focused and divided attention in each half of space with disconnected hemispheres. Cortex, 25, 33-45.

\section{NOTES}

1. Since the original formulation of the variable-criterion theory (Grice, 1968), more elaborate versions of the model have been devised (e.g., Grice, Nullmeyer, \& Schnizlein, 1979; Grice, Nullmeyer, \& Spiker, 1982) in which information does not accumulate linearly from a fixed intercept. However, because these alternative models do not add information to the problem under investigation and because they require elaborate mathematical expressions that burden the treatise, they will not be dealt with in the present manuscript.

2 . An interesting analogy that may help one to understand the meaning of the different aspects of the model is that of gambling on a horse race. The time between the moment of betting and the moment of winning (or losing) is a function of a number of components. First, there are components unrelated to the race $\left(R_{0}\right)$, and, second, there are characteristics of the race itself. On the average, the race will take longer the fewer the horses that start, the slower the horses are, the longer the distance is, and the more the horses that must reach the finish before one knows whether one has won. The same principles hold for information transfer in the human brain. A large visual stimulus will excite more receptors. Because of the large number of cells excited, more fast conducting fibers will be among them and information accumulation will grow more rapidly. The time to surpass the detection criterion, then, is a function of the distance that the signals have to travel and the number of impulses that must be added before the threshold is reached.

3. In the signal detection literature, there is some discussion of whether variation of the proportion of catch trials only affects the criterion location and not the sensitivity (e.g., Dusoir, 1983). Within the framework of the variable criterion theory, this can be tested by looking at the response evocation characteristics (RECs, Grice et al., 1979; Grice et al., 1982) of the growth functions derived from the cumulative RT distributions. If the proportion of catch trials does not alter sensitivity, (1) the functions for the different criteria should be linear transformations of each other, and (2) the parameters of the linear models should be the same for all four stimulus intensities. Both requirements were met: Based on the smooth group distributions (combined over crossed and uncrossed conditions), the percentage of variance explained by linear relationship ranged from $R^{2}=.995$ to $R^{2}=$ .999 , and the differences between the estimates for the four stimulus intensities were not larger than expected by chance. In addition, the slopes of all RECs fluctuated around unity, indicating that the standard deviations for the three criterion levels were identical.

4. It may be interesting to note that across the studies listed by Marzi et al. (1991), there was no significant correlation between the IHTT estimate and the mean RT of the crossed and the uncrossed conditions $(r=.172, n=15$; the study of Moscovitch and Smith, 1979, was not included in our analysis because it is a SD study; see Table 1).

5 . One of these two studies was an experiment by Bashore and colleagues, mentioned in a footnote of Bashore (1981). Because no details of the study were given, it has not been retained in Table 1 of the present paper.

6. The model has been applied to the data of Sergent and Myers (1985) as well, but it did not return the same clear-cut results as did the one based on the findings of Clarke and Zaidel (1989), even though the same patients were tested. This has probably to do with the fact that Sergent and Myers based their results on only 100 observations. The same was true for the verbal IHTT measures of Sergent and Myers.

7. As in note 3, RECs confirmed the viability of the assumption that the introduction of catch trials only affected the decision criterion and not the sensitivity. Functions based on the group data for the different percentages of catch trials were linearly related to one another $(.996 \leq$ $R^{2} \leq .999$ ), and the parameter estimates were comparable for all four stimulus intensities. In addition, the analysis showed that the standard deviations of the criterion levels were similar for the two conditions with catch trials and about $15 \%$ larger than the standard deviation for the SRT condition.

(Manuscript received February 18, 1993; revision accepted for publication April 11, 1994.) 